Prepared for Publication as an Article in Synthetic Metals

\title{
Protection of 2-(3-Thienyl)ethanol with 3-Thienylacetic Acid and Hard Cross- Linked Conducting Films by Electropolymerization of the Ester
}

\author{
Amala Dass ${ }^{1}$, Sudir Mulik ${ }^{1}$, Chariklia Sotiriou-Leventis ${ }^{1, *}$ and Nicholas Leventis ${ }^{2, *}$ \\ 1. Department of Chemistry, University of Missouri-Rolla, Rolla, MO 65409 \\ 2. Materials Division, NASA Glenn Research Center, 21000 Brookpark Road, \\ M.S. 49-1, Cleveland, OH 44135.
}

\begin{abstract}
The ester (compound 1) of 2-(3-thienyl)ethanol (T-etOH) with 3-thienylacetic acid was synthesized as a monomer whose two thiophene groups could be electropolymerized independently, becoming members of different polymer chains in a highly-crosslinked highly-insoluble polymer. Indeed, 1 was electropolymerized successfully alone and together with 3-methylthiophene (3MeT). Films of poly(1) are hard $(3 \mathrm{H}$, as opposed to $<6 \mathrm{~B}$ for poly $(\mathbf{3 M e T}))$, and the close proximity of the polymeric strands creates $\pi$-stacking interactions. The behavior of 1 suggests that by: (a) limiting the potential used for the oxidation of monomeric esters of T-etOH at the foot of their oxidation waves $(<1.8 \mathrm{~V}$ vs. $\mathrm{Ag} / \mathrm{AgCl})$; and, (b) compensating for the decrease in the electrogenerated radical concentration by increasing the monomer concentration, practically all esters of T-etOH should be electropolymerizable. This was confirmed by durable film formation from the archetypical ester of T-etOH, the 2-(3-thienyl)ethyl acetate (T-etOAc), whose homo-electropolymerization is reported for the first time.
\end{abstract}

Keywords: electropolymerization, cross-linked, ester, 2-(3-thienyl)ethanol

* Corresponding authors: Tel.: +1 5733414353 (C. S.-L.), +1 2164333202 (N. L.) E-mail addresses: cslevent@umr.edu (C. S.-L.), Nicholas.Leventis@ nasa.gov (N. L.). 


\section{Introduction}

Invariably, microdevices designed to carry out useful function based on the electrical conductivity of 'conducting' polymers (e.g., polypyrrole, polyaniline, polythiophene etc.) incorporate two closely-spaced microelectrodes bridged by the polymer. ${ }^{1}$ This configuration, produced by electrodeposition of the conducting polymer, was introduced by Wrighton in the mid 1980's, ${ }^{2}$ and is an extremely convenient fabrication approach, ${ }^{3}$ because it is fast, directional to the place where polymer must be deposited, utilizes small amounts of monomer, and most importantly, it relies on a single "generic" substrate. On the other hand, for most practical purposes, the monomer precursor of the conducting polymer should carry functionality that would not interfere with electropolymerization, and eventually could be used for attachment of, for example, biologically active moieties leading to biorecognition and biosensors. Among possible conducting polymers, poly(aniline) is conducting only from its two-electron oxidized, doubly-protonated form (emeraldine salt) and therefore the solution $\mathrm{pH}$ should be controlled closely, ${ }^{4}$ poly (pyrroles) have limited shelf-life, ${ }^{5}$ while poly(thiophenes) appear the most versatile. ${ }^{6}$ In this context, 3 -substituted thiophenes not only avoid interference with the desirable $\alpha, \alpha^{\prime}$ coupling, but also minimize $\alpha, \beta^{\prime}$ couplings that compromise the conductivity of the polymer. ${ }^{6}$ With regards to the chemical identity of possible substituents, again one would prefer a single reliable functional group, capable of reacting and coupling with a variety of modifiers. That polymer could be based on 2-(3thienyl)ethanol (T-etOH), whose short tether combines minimal steric requirements with

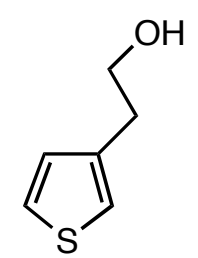

T-etOH

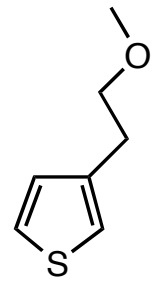

T-etOMe

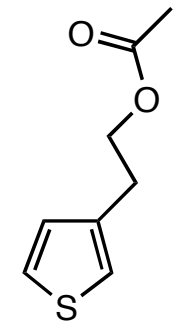

T-etOAc

a reasonable attenuation of the electronic effect of the hydroxyl group on the thiophene ring. ${ }^{7}$ Numerous reports, however, indicate that T-etOH cannot be electropolymerized, ${ }^{7}$ presumably because the oxidized form is prone to nucleophylic attack at the 3- and 4positions, ${ }^{8}$ where the role of the nucleophile can be played by another monomer, leading 
to intractable non-electroactive coupling products. ${ }^{9}$ Of course, the alternative is to protect the $-\mathrm{OH}$ group through etherification or esterification. That possibility has been investigated extensively, ${ }^{7}$ and it has been found that in general, ethers of T-etOH lead to passivation of the electrode, while reportedly only 3-(2-methoxyethyl)thiophene (TetOMe) has been electropolymerized successfully. ${ }^{7 a}$ On the other hand, as far as esters of T-etOH are concerned, only co-polymers with 3-methylthiophene (3MeT) are known. ${ }^{7 b}$ The lack of homopolymerization of esters of $\mathbf{T}$-etOH has been attributed to relatively high oxidation potentials ( $>2.1 \mathrm{~V}$ vs. $\mathrm{Ag} / \mathrm{AgCl}$ ), which presumably cause competition between polymerization and oxidative decomposition (referred to as overoxidation). Indeed, longer tethers between the thiophene system and an ester or even a carboxyl group seem to attenuate the electronic effects of the substituent, and, for example, 4nitrophenyl-11-(3-thienyl)undecanecarboxylate, or 3-(4-carboxybutyl)thiophene have been electropolymerized uneventfully. ${ }^{10}$ In another approach, ${ }^{11}$ synthesis of bi- and trithiophene analogues of T-etOH yields monomeric esters with more extended $\pi$-systems and much lower oxidation potentials ( $\mathrm{E}^{\mathrm{o}} \sim 1.2 \mathrm{~V}$ vs. $\mathrm{Ag} / \mathrm{AgCl}$ for dimers, ${ }^{11 \mathrm{a}, \mathrm{e}}$ and $\mathrm{E}^{\mathrm{o}}<0.9 \mathrm{~V}$ vs. $\mathrm{Ag} / \mathrm{AgCl}$ for trimers ${ }^{11 \mathrm{c}, \mathrm{d}}$ ) than those reported for typical esters of T-etOH such as, for example, 2-(3-thienyl)ethyl acetate (T-etOAc, $\mathrm{E}^{\mathrm{o}} 2.49 \mathrm{~V}$ vs. $\left.\mathrm{Ag} / \mathrm{AgCl}\right){ }^{7 \mathrm{~b}}$

Nevertheless, it also came to our attention that although voltammograms of esters of T-etOH lack reduction waves, in general, they do not seem to passivate the electrode either: reportedly, those esters can be oxidized continuously, producing violet streaks that disappear upon reduction. ${ }^{7 b}$ Thus, it seemed to us that in addition to irreversible decomposition, ${ }^{12}$ perhaps lack of homopolymerization into redox-active films is also due partly to formation of soluble oligomers. In order to decrease the solubility of the developing polymer we decided to introduce crosslinking through $\beta, \beta^{\prime}$-type coupling that would not affect the film conductivity. ${ }^{6}$ For this purpose we prepared 2-(3-thienyl)ethyl3-thienyl acetate (1), where the hydroxyl group of T-etOH is protected by esterification

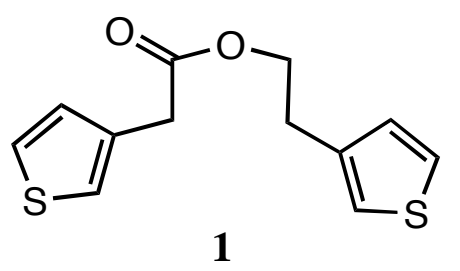


with 3-thienylacetic acid. The central idea in the design of $\mathbf{1}$ was that since both models for its two halves, namely T-etOAc and methyl-3-thiophene acetate, are oxidizable in the same potential range $(>1.7 \mathrm{~V}$ vs. $\mathrm{Ag} / \mathrm{AgCl}){ }^{7 b, 13}$ we should expect that both thiophene groups should be electropolymerizable simultaneously becoming parts of different polymer chains in a highly crosslinked, highly insoluble polymer. Furthermore, it also seemed reasonable that the high insolubility of the developing polymer would make possible to induce film formation even with relatively low radical concentrations, namely working at the foot of the monomer oxidation wave $(<1.8 \mathrm{~V}$ vs. $\mathrm{Ag} / \mathrm{AgCl})$, thus avoiding overoxidation. Indeed, this working hypothesis has been fully confirmed, and we have been able to not only polymerize 1 both by itself [to poly(1)] and together with 3 MeT [to copoly(1-3MeT)], but also to utilize the developing expertise to the electropolymerization of T-etOAc and demonstrate that contrary to previous reports it can be also homoelectropolymerized into durable films by: (a) keeping the applied potential low; and, (b) using higher monomer concentrations (0.2 M). Films were characterized electrochemically, spectroelectrochemically, as well as by specularreflectance infrared spectroscopy. Film conductivity was measured using home-made interdigitated microelectrode arrays (IDAs).

\section{Experimental Section}

Materials. All reagents and solvents were used as received unless otherwise noted. Anhydrous acetonitrile, 3-methylthiophene (3MeT), 3- thienylacetic acid (98\%), 2-(3-thienyl)ethanol (T-etOH) (99\%), and thionyl chloride (97\%) were purchased from Aldrich Chemical Co. Triethylamine $\left(\mathrm{Et}_{3} \mathrm{~N}\right)$ was purchased from Acros Chemicals and was further purified by distillation from calcium hydride. Tetrabutylammonium perchlorate (TBAP) was prepared from an aqueous solution of tetrabutylammonium bromide (Aldrich) and $70 \%$ aqueous $\mathrm{HClO}_{4}$ as described before. ${ }^{14}$

Synthesis of 1. A solution of 3-thiopheneacetic acid $5.16 \mathrm{~g}(\sim 0.0354 \mathrm{~mol})$ in thionyl chloride $(16 \mathrm{ml})$ was refluxed for $1.5 \mathrm{~h}$ while protected from moisture with a drying tube. Excess thionyl chloride was removed under reduced pressure and the residue was dried for $4 \mathrm{~h}$ in a vacuum oven $\left(40{ }^{0} \mathrm{C}\right)$ to remove traces of remaining thionyl chloride. To the residue in anhydrous $\mathrm{CH}_{2} \mathrm{Cl}_{2}(44 \mathrm{ml})$ was added $\mathbf{T}$-etOH $(4 \mathrm{ml}, 0.0354 \mathrm{~mol})$ followed by 
$\mathrm{Et}_{3} \mathrm{~N}(4.9 \mathrm{ml}, 0.0354 \mathrm{~mol})$ and the reaction mixture was stirred at room temperature for $24 \mathrm{~h}$. At the end of the period, the reaction mixture was extracted with water $(3 \times 40 \mathrm{ml})$, the organic layer was dried with anhydrous $\mathrm{Na}_{2} \mathrm{SO}_{4}$ and evaporated to dryness. The crude product was purified on a silica gel column using $\mathrm{CH}_{2} \mathrm{Cl}_{2} / \mathrm{CH}_{3} \mathrm{OH}(99: 1, \mathrm{v} / \mathrm{v})$ as eluent. Further purification by vacuum distillation, gave a pale yellow liquid; b.p. $141-152^{\circ} \mathrm{C}$ at 0.25 torr; yield: $6.8147 \mathrm{~g}(76.28 \%)$. ${ }^{1} \mathrm{H}$ NMR $\delta$ 6.91-7.28 (6 H, m, Ar), 4.32 (t, $2 \mathrm{H}, J=$ $6.8 \mathrm{~Hz}, \mathrm{CH}_{2} \mathrm{O}$ ), 3.65 (s, $2 \mathrm{H}, \mathrm{CH}_{2} \mathrm{C}=\mathrm{O}$ ), $2.96\left(\mathrm{t}, 2 \mathrm{H}, J=6.8 \mathrm{~Hz}, \mathrm{CH}_{2}\right) ;{ }^{13} \mathrm{C} \mathrm{NMR} \delta 171.0$, $137.8,133.5,128.4,128.2,125.7,125.6,122.8,121.6,64.7,35.9,29.5$; IR $\vee 3103(\mathrm{~m})$, 2957 (m), 1734 (s), 1536 (w), 1411 (m), 1260 (s), 1147 (s), 1081(w), 1009 (m), 945 (w), 859 (m), $833(\mathrm{~m}), 774$ (s), $689(\mathrm{~m}) \mathrm{cm}^{-1}$; Anal. Calcd for $\mathrm{C}_{12} \mathrm{H}_{12} \mathrm{~S}_{2} \mathrm{O}_{2}: \mathrm{C}, 57.12 ; \mathrm{H}, 4.79$; S, 25.41. Found: C, 57.01, H, 5.05, S, 24.82.

Instrumentation and Methods. Polymer electrodeposition and standard electrochemical characterization were conducted with an EG\&G 263A potentiostat controlled by the EG\&G model 270/250 Research Electrochemistry Software 4.30. Characterization of the film transconductance was conducted using interdigitated microelectrode arrays (IDAs) and a PINEAFRDE5 Bipotentiostat (Pine Instruments); data were captured on computer using the EG\&G model 270/250 software and were recorded simultaneously on a Kipp and Zonen $\mathrm{X}-\mathrm{Y}-\mathrm{Y}^{\prime}$ recorder. Gold and platinum disk electrodes $\left(2.0 \mathrm{~mm}\right.$ diameter, $\left.0.0314 \mathrm{~cm}^{2}\right)$ and $\mathrm{Ag} / \mathrm{AgCl} /$ aqueous $\mathrm{KCl}(3 \mathrm{M})$ reference electrodes were purchased from $\mathrm{CH}$ Instruments, Inc. (Austin, TX). The counter electrode was constructed from a Pt mesh (Aldrich). Disk working electrodes were polished successively with 6,3 , and $1 \mu \mathrm{m}$ diamond paste (Struers Inc., Westlake, $\mathrm{OH}$ ), washed with water and acetone, and air-dried. Chromium/gold-coated glass slides were used as working electrodes for depositing films for specular reflectance infrared analysis, and were prepared using microscope slides and a Baltek MED 20 sputtering system. Indium-tin-oxide (ITO) coated glass slides for depositing films for UV-Vis. spectroelectrochemistry were available from previous work. ${ }^{15}$ Home-made IDAs used for transconductance work consist of 100 microelectrodes (50 on each side), $0.4 \mathrm{~mm}$ long, 3 $\mu \mathrm{m}$ wide and $3 \mu \mathrm{m}$ apart from one another, and were made photolithographically at the Microfabrication lab at NASA Glenn Research Center from chrome masks prepared by Advanced Reproductions, Inc. (North Andover, MA). All volumetric glassware was 
rinsed with acetone, then water, then soaked in a Micro cleaning solution in water, rinsed with copious amounts of water, and oven dried overnight.

Fourier-Transformed Infrared (FT-IR) spectra were obtained with a Nicolet Nexus 470 spectrophotometer. Grazing angle studies were performed at $80^{\circ}$ using a specular reflectance attachment Model 500 (Thermo Electron Co.). UV-Vis spectroelectrochemistry was conducted with an Ocean Optics, Inc., Model CHEM2000 miniature fiber optic spectrophotometer. Energy Dispersive Analysis of X-rays (EDAX) was conducted with a Hitachi S4700 FESEM instrument using a $4 \mathrm{keV}$ electron beam and an EDAX energy dispersive X-ray detector calibrated with aluminum and chromium. Film hardness was determined according to ASTM D3363-00 Standard Test Method for Film Hardness by Pencil Test.

Electropolymerization of $\mathbf{1}$ was performed either potentiostatically or voltammetrically from $\mathrm{CH}_{3} \mathrm{CN} / 0.5 \mathrm{M}$ TBAP solutions. The upper potential limit (1.75 V vs. aq. $\mathrm{Ag} / \mathrm{AgCl}$ ) was optimized to avoid over-oxidation. Co-polymerizations were performed either with a 2:1 or a 1:1 mol ratio of $\mathbf{1}$ and $\mathbf{3 M e T}$ in anhydrous $\mathrm{CH}_{3} \mathrm{CN} / 0.5 \mathrm{M}$ TBAP. The electrode potential was scanned from 0 to $1.8 \mathrm{~V}$ vs. aq. $\mathrm{Ag} / \mathrm{AgCl}$ at $0.1 \mathrm{~V} \mathrm{~s}^{-1}$.

\section{Results and Discussion}

\subsection{Electrochemical homopolymerization of 2-(3-thienyl)ethyl esters. Although} esters of T-etOH have not been electropolymerized before, it was noted, however, that electropolymerization attempts involved very positive potentials ( $>2 \mathrm{~V}$ vs. $\mathrm{Ag} / \mathrm{AgCl})$, while oxidation of the monomers starts as low as $1.7 \mathrm{~V}$ vs. $\mathrm{Ag} / \mathrm{AgCl} .^{7 \mathrm{~b}}$ Considering that observation together with the fact that oxidation of those esters does not lead to any film formation, but rather to what appears to be soluble oligomers (see Introduction), we decided to increase the chances for film formation by: (a) synthesizing monomer 1 that would result in a crosslinked polymer, which presumably would be very insoluble; (b) limiting the potential range of electrodeposition; and, (c) compensating for the lower concentration of radicals by increasing the monomer concentration.

Monomer 1 was prepared by a straight-forward esterification of 2-(3thienyl)ethanol with 3-thiopheneactyl chloride (Scheme 1). 
Scheme 1. Preparation of 1.

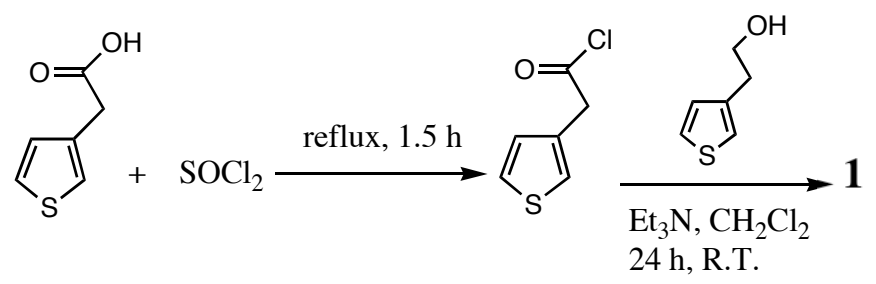

Electropolymerization of 1 was first carried out in a $\mathrm{CH}_{3} \mathrm{CN} / 0.5 \mathrm{M}$ TBAP solution containing $0.2 \mathrm{M}$ of 1 . Au and Pt disk electrodes were swept once from zero to $1.75 \mathrm{~V}$ vs. $\mathrm{Ag} / \mathrm{AgCl}$ at $0.1 \mathrm{~V} \mathrm{~s}^{-1}$ (Figure $1 \mathrm{~A}-$ single sweep). It is noted that the voltammertic wave is irreversible, the return trace crosses the forward sweep indicating film formation, ${ }^{13,16}$ and the appearance of a reduction wave with peak current at around $+1.0 \mathrm{~V}$ vs. $\mathrm{Ag} / \mathrm{AgCl}$ implies that the electrodeposited film is redox-active. It is noted further that potentiostatic electropolymerization of $\mathbf{1}$ at $1.75 \mathrm{~V}$ vs. $\mathrm{Ag} / \mathrm{AgCl}$ using lower concentrations of the monomer (as low as $0.01 \mathrm{M}$ ) was also successful. Overall, it seems that the monomer concentration is not a critical factor for film formation from $\mathbf{1}$. This, combined with the fact that electrodeposition of films of poly(T-etOAc) from $0.05 \mathrm{M}$ solutions of the monomer has not been successful before ${ }^{7 \mathrm{~b}}$ implies that the high degree of crosslinking expected from $\mathbf{1}$ does play an important role in film formation. In all cases, electrodes were coated with golden-yellow films of poly(1), which are redox-active (Figure 1B) showing the typical shape of conducting polythiophene-type films. ${ }^{6}$ The prepeak observed in the anodic wave is attributed to the familiar stereoelectronic coupling in the doped-state of conducting polymers, as well as to the conformational changes taking place as the polymeric backbone gets oxidized. ${ }^{17,6,10 \mathrm{c}}$ The electrode coverage (Figure 1legend) was calculated from the area under the voltammetric wave and it can vary depending on the number of voltammetric sweeps or the time allowed after a potential step. Films of poly(1) are uniform (<50 nm minimum feature-size), compact (Figure 2) and hard; their hardness, evaluated with a standard pencil test, was found equal to $3 \mathrm{H}$, and is much higher than the value of less than 6B given by poly(3MeT) films.

The ease of electropolymerization of $\mathbf{1}$ as well as the electrochemical stability of the resulting films prompted us to re-examine whether T-etOAc itself could yield redoxactive films. Our attempts were successful by using higher concentrations of the 
monomer (e.g., 0.2 M) in combination with less oxidizing potentials. The voltammogram of T-etOAc (Figure 1C - multiple sweeps) shows similar features to those demonstrated by $\mathbf{1}$, and indeed $\mathrm{Au}$ or Pt disk electrodes get covered with gold-yellow redox-active films (Figure 1D) just like in the case of 1. The higher oxidation potential of poly(1) (oxidation starts at $\sim 0.9 \mathrm{~V}$ vs. $\mathrm{Ag} / \mathrm{AgCl}$ ) relative to poly(T-etOAc) (oxidation starts at $\sim 0.7 \mathrm{~V}$ vs. $\mathrm{Ag} / \mathrm{AgCl}$ ) is attributed to different electron withdrawing properties of the methyl and the 3-thienylmethyl groups.

Subsequently, films of poly(1) and of poly(T-etOAc) were deposited on $\mathrm{Cr} / \mathrm{Au}-$ and ITO-coated glass slides by both a potential sweep and a potential step method, and were characterized by specular reflectance infrared and by UV-Vis absorption spectroscopy. The dominant feature in the IR spectra is the stretching vibration of the ester at $1749 \mathrm{~cm}^{-1}$ and at $1753 \mathrm{~cm}^{-1}$ for poly(1) and poly(T-OAc), respectively (Figures $3 \mathrm{~A}$ and $3 \mathrm{~B})$. On the other hand, the electronic absorption spectra of the two films (Figures 3C and 3D) show absorptions at 421 and $432 \mathrm{~nm}$ respectively, while their oxidized forms appear blue and both absorb above $700 \mathrm{~nm}(716 \mathrm{~nm}$ and $769 \mathrm{~nm}$, respectively), in analogy to the characteristic polaronic absorption of poly(3MeT) at 774 nm. ${ }^{6}$ The blue shift in the absorption spectrum of oxidized poly(1) relative to the spectrum of the oxidized form of poly(T-OAc) is consistent with the stabilization of the HOMO reflected by the higher oxidation potential of poly(1). A second noteworthy feature in the absorption spectrum of the oxidized form of poly(1) is the short wavelength shoulder at $604 \mathrm{~nm}$. That absorption is developing progressively as the degree of the film oxidation increases, but it is not a bipolaronic absorption as it falls at shorter wavelengths than the polaronic absorption of poly(1) at $716 \mathrm{~nm}$. That feature is attributed to stabilization through $\pi$-system interactions of neighboring polarons in adjacent polythiophene chains, ${ }^{18}$ in analogy to the classic $\pi$-interactions observed in millimolar solutions ( $\pi$-stacking dimerization) and in films of long-wavelength absorbing cationic dyes, such as methylene blue. ${ }^{19}$ Indeed, the stabilization energy of that interaction (calculated from the energy difference between $604 \mathrm{~nm}$ and $716 \mathrm{~nm}$ ) is $0.32 \mathrm{eV}$, and corresponds to the range for exciton-exciton interactions leading to the so-called Davydov blue shift. ${ }^{18 d}$ Finally, the fact that the $\pi$-system stabilization is so pronounced in the case of poly(1) probably reflects the effect of the ester bridges holding the polymer 
chains within less than $4 \AA$ from one another, ${ }^{18 \mathrm{c}}$ a fact which should facilitate interchain electron hoping and should be reflected in the polymer conductivity. From a practical perspective, spectroelectrochemistry agrees well with voltammetry in the sense that oxidation of poly(T-etOAc) starts $\sim 0.3 \mathrm{~V}$ before oxidation of poly(1), while, depending on the polymer, polaronic absorptions eventually decline irreversibly above 1.3-1.6 V vs. $\mathrm{Ag} / \mathrm{AgCl}$, presumably owing to overoxidation.

\subsection{Copolymerization of 1 with 3-methylthiophene, and film conductivity.} Although co-polymerization of esters of T-etOH with 3-methylthiophene (3MeT) as a means of introducing hydroxyl functionality in polythiophene chains is no longer necessary, nevertheless, co-polymerization of $\mathbf{1}$ with $\mathbf{3 M e T}$ is still important as a means of "diluting" the hydroxyl functionality, particularly for applications where subsequent derivatization with bulky reagents may lead to over-crowding and retardation of the ionic flux in and out of the film that is necessary for redox-switching. ${ }^{20}$ Figure $4 \mathrm{~A}$ shows the voltammogram of a $\mathrm{CH}_{3} \mathrm{CN} / 0.5 \mathrm{M}$ TBAP solution that contains $0.2 \mathrm{M}$ of 1 and $0.1 \mathrm{M}$ 3MeT. Again, for reasons outlined above, the potential sweep was kept well below the reported range for the peak-current potentials for this class of compounds $(>2.1 \mathrm{~V}$ vs. $\mathrm{Ag} / \mathrm{AgCl}$ ), and again we observe both a crossing of the forward and return traces, and a cathodic wave centered at $\sim 1.0 \mathrm{~V}$ vs. $\mathrm{Ag} / \mathrm{AgCl}$, as it was observed during homopolymerization of 1 . The yellow-orange film of copoly(1-3MeT) are compact showing hardness values $(\mathrm{H})$ intermediate between those of poly(1) (3H) and poly(3MeT) (<6B). Voltammetrically, (Figure 4B) the redox wave of copoly(1-3MeT) falls between the waves of poly(3MeT) and poly(1) pointing to a polymer that incorporates both monomers, as opposed to a blend of two polymers. The dominant feature in the IR spectrum of copoly(1-3MeT) is the carbonyl absorbance at $1736 \mathrm{~cm}^{-1}$ (Figure 5A), confirming the incorporation of 1. Energy Dispersive Analysis of X-rays (EDAX, Figure 5B) based on relative signal intensities gives an atomic S/O ratio of 1.0796, which corresponds to a 1-to-3MeT ratio of 6.3.

Film conductivities were followed through their transconductance using homemade interdigitated microelectrode arrays (IDAs) connected in the conventional transistor mode (Figure 6A). ${ }^{2}$ Owing to the film compactness, we have not been able to bridge the 3 
$\mu \mathrm{m}$ gap between the microelectrodes of our arrays with poly(1). We have been able to do so, however, with films of copoly(1-3MeT) and poly(T-etOAc). Figure 6B shows a photograph of actual IDA microelectrodes coated with copoly(1-3MeT), while the inset shows the entire IDA device. The potential the polymer is held at (here called gate voltage, $\left.\mathrm{V}_{\mathrm{G}}\right)$ is swept at $0.01 \mathrm{~V} \mathrm{~s}^{-1}$ from a reducing potential $(0.0 \mathrm{~V}$ vs. $\mathrm{Ag} / \mathrm{AgCl})$ to an oxidizing one $(1.225 \mathrm{~V}$ vs. $\mathrm{Ag} / \mathrm{AgCl})$ while we monitor the drain current $\left(\mathrm{I}_{\mathrm{D}}\right)$ that flows between the IDA microelectrodes as a result of a constant drain voltage $\left(V_{D}=20 \mathrm{mV}\right)$ applied across them. The resulting transconductance curves $\left(I_{D}\right.$ vs. $\left.V_{G}\right)$ are shown in Figure 7 in comparison with a typical curve obtained with poly(3MeT). At low gate voltages no drain current flows between the microelectrodes of the IDA in response to the $20 \mathrm{mV}$ drain bias, because the polymers in their reduced forms are electrical insulators. ${ }^{2 a}$ As the polymers become oxidized, drain current $\left(\mathrm{I}_{\mathrm{D}}\right)$ starts flowing through the electrodes of the IDA. Consistently with the voltammetric results, the potential range in which copoly(1-3MeT) becomes conducting is more positive than the corresponding range of poly(3MeT), but about the same $I_{D}$ current flows though both polymers in their conducting states $(\sim 1.5 \mathrm{~mA})$. On the other hand, films of poly(T-etOAc) appear significantly less conducting. The high conductivity of poly(3MeT) is associated with $\alpha, \alpha^{\prime}$ couplings in combination with a favorable polymeric conformation that allows substantial orbital overlap between adjacent monomer units. ${ }^{6,21}$ However, as Miller has pointed out, ${ }^{18 c}$ the conductivity of conducting polymers is a three-dimensional issue, meaning that efficient inter-polymer-chain electron hopping is a necessity for obtaining good conductivity. ${ }^{22,23}$ Thus, it is reasonable to assume either that the conformations of copoly(1-3MeT) and poly(3MeT) are very similar, or most probably that the ester linkages hold the polymer chains close together compensating for possible conductivity losses due to compromised orbital overlap.

\section{Conclusions}

Overoxidation has been avoided and esters of T-etOH have been electropolymerized by inducing oxidation at the foot of the monomer oxidation waves $(<1.8 \mathrm{~V}$ vs. $\mathrm{Ag} / \mathrm{AgCl})$, while at the same time the monomer concentration has been increased in order to compensate for the decrease in the electrogenerated radical 
concentration. This strategy was demonstrated by homoelectropolymerization of 2-(3thienyl)ethyl acetate (T-etOAc), while using the ester functionality in order to cross-link independently growing polythiophene chains (compound 1) yields hard, highly insoluble films.

Acknowledgement. The authors thank the NASA-GRC Independent Research and Development Fund for financial support, Mrs. Elizabeth A. McQuaid for microfabrication of IDAs, Mrs. Linda S. McCorkle for SEM and Mr. Terry R. McCue for EDAX. 


\section{Figure Captions}

Figure 1. Cyclic voltammetry in Ar-degassed solutions with a Au disk electrode (2.0 mm diameter) at $0.1 \mathrm{~V} \mathrm{~s}^{-1}$. A and $\mathrm{C}$ : Voltammograms of $\mathbf{1}(0.2 \mathrm{M}$, single sweep) and of T-etOAc (0.2 M, three sweeps), respectively, in $\mathrm{CH}_{3} \mathrm{CN} / 0.5 \mathrm{M}$ TBAP. B and C: Voltammetry of the corresponding polymer films in $\mathrm{CH}_{3} \mathrm{CN} / 0.1 \mathrm{M}$ TBAP. Coverage: poly(1), $1.31 \mathrm{mC} \mathrm{cm}$; poly(T-etOAc), $12.6 \mathrm{mC} \mathrm{cm}^{-2}$. (Numbers shows successive potential sweeps.)

Figure 2. A Scanning Electron Micrograph (SEM) of poly(1) electrodeposited on a $\mathrm{Cr} / \mathrm{Au}$ sputtered glass slide. Inset: profilometry across two trenches scratched in the film (made potentiostatically, coverage: $1.69 \mathrm{mC} \mathrm{cm}^{-2}$ ).

Figure 3. A, B: Specular reflectance IR of poly(1) and of poly(T-etOAc) films, respectively, on $\mathrm{Cr} / \mathrm{Au}$-coated glass slides. C, D: Spectroelectrochemical data from the same polymers on ITO-coated glass slides in $\mathrm{CH}_{3} \mathrm{CN} / 0.1 \mathrm{M}$ TBAP.

Figure 4. Cyclic voltammetry (CV) in Ar-degassed solutions with a Au disk electrode (2.1 mm diameter) at $0.1 \mathrm{~V} \mathrm{~s}^{-1}$. A: CV of a mixture of $\mathbf{1}(0.2 \mathrm{M})$ and 3MeT $(0.1$ $\mathrm{M})$ in $\mathrm{CH}_{3} \mathrm{CN} / 0.5 \mathrm{M}$ TBAP. B: $\mathrm{CV}$ of the resulting copoly(1-3MeT) in comparison with the voltamograms of poly(3MeT) and of poly(1). (Coverage of copoly(1-3MeT): 5.49 $\mathrm{mC} \mathrm{cm} \mathrm{cm}^{-2}$ )

Figure 5. Specular reflectance IR spectrum (top) and EDAX spectrum (4 $\mathrm{kV}$, bottom) of copoly(1-3MeT) on $\mathrm{Cr} / \mathrm{Au}$ sputtered glass slides. (The black trace in the EDAX spectrum is the background from a Au-coated glass slide.)

Figure 6. A: Circuitry for monitoring the conductivity of a redox-active polymer as a function of the electrode potential (gate voltage: $\mathrm{V}_{\mathrm{G}}$ ). B: Optical photograph of an interdigitated electrode array (IDA) coated with copoly(1-3MeT). Inset in B: the device carrying the array and its contacting pads, and a detail of a clean array. 
Figure 7. Transconductance curves of polymer films electrodeposited on IDAs, as the electrode potential $\left(\mathrm{V}_{\mathrm{G}}\right.$, refer to Figure $\left.6 \mathrm{~A}\right)$ is varied at $0.01 \mathrm{~V} \mathrm{~s}^{-1}$ from a range where the polymers are in their reduced/insulating states $(<0.5 \mathrm{~V}$ vs. $\mathrm{Ag} / \mathrm{AgCl})$, to the potential range where they are oxidized and electrically conducting. The electrical conductivity is proportional to the drain current $\left(\mathrm{I}_{\mathrm{D}}\right)$ flowing through the polymer films. 


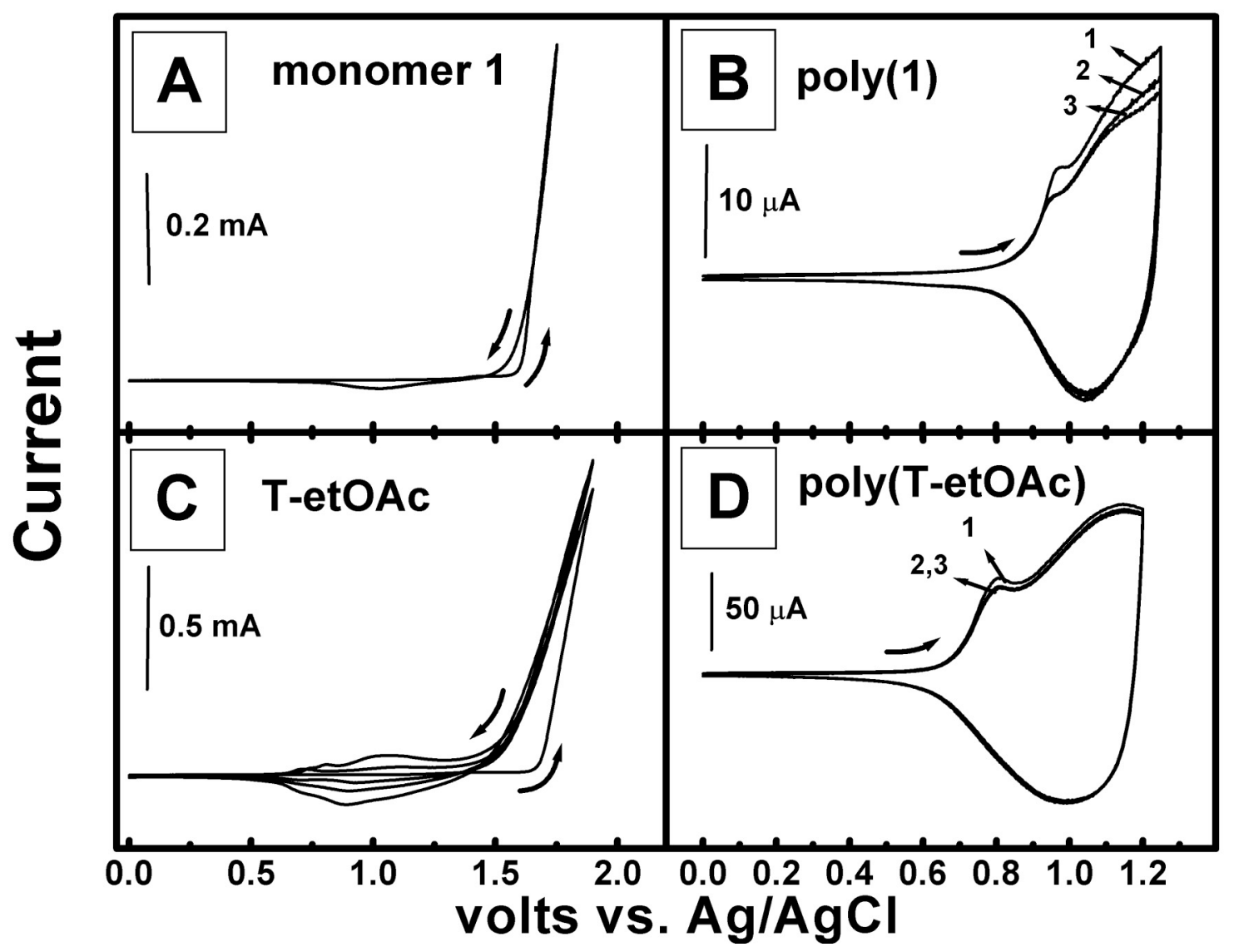

Figure 1. 


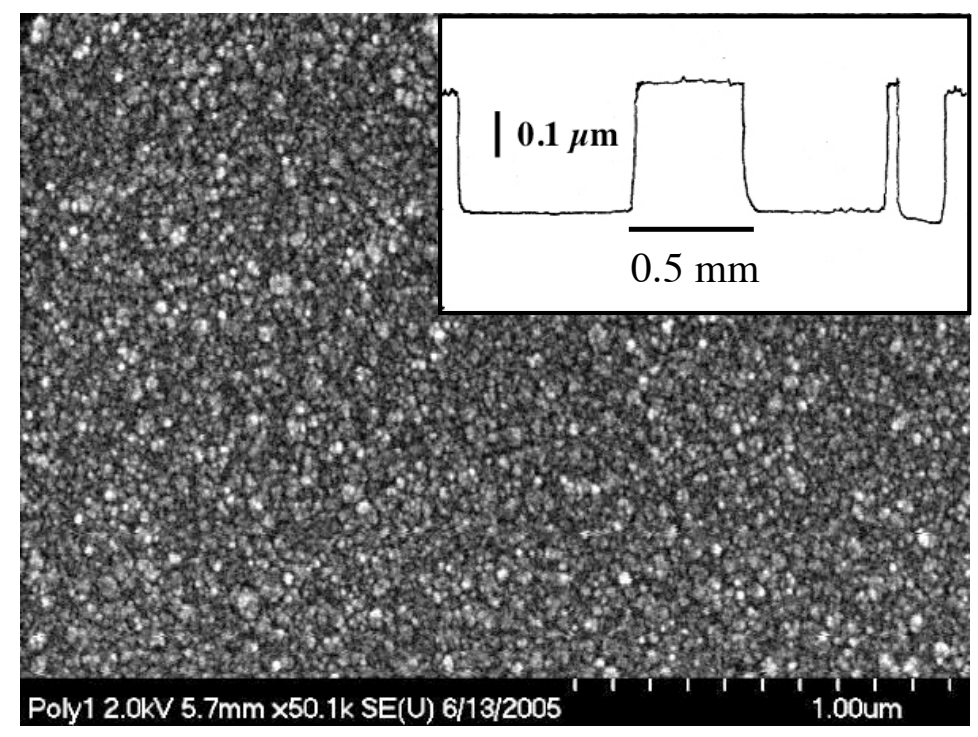

Figure 2. 


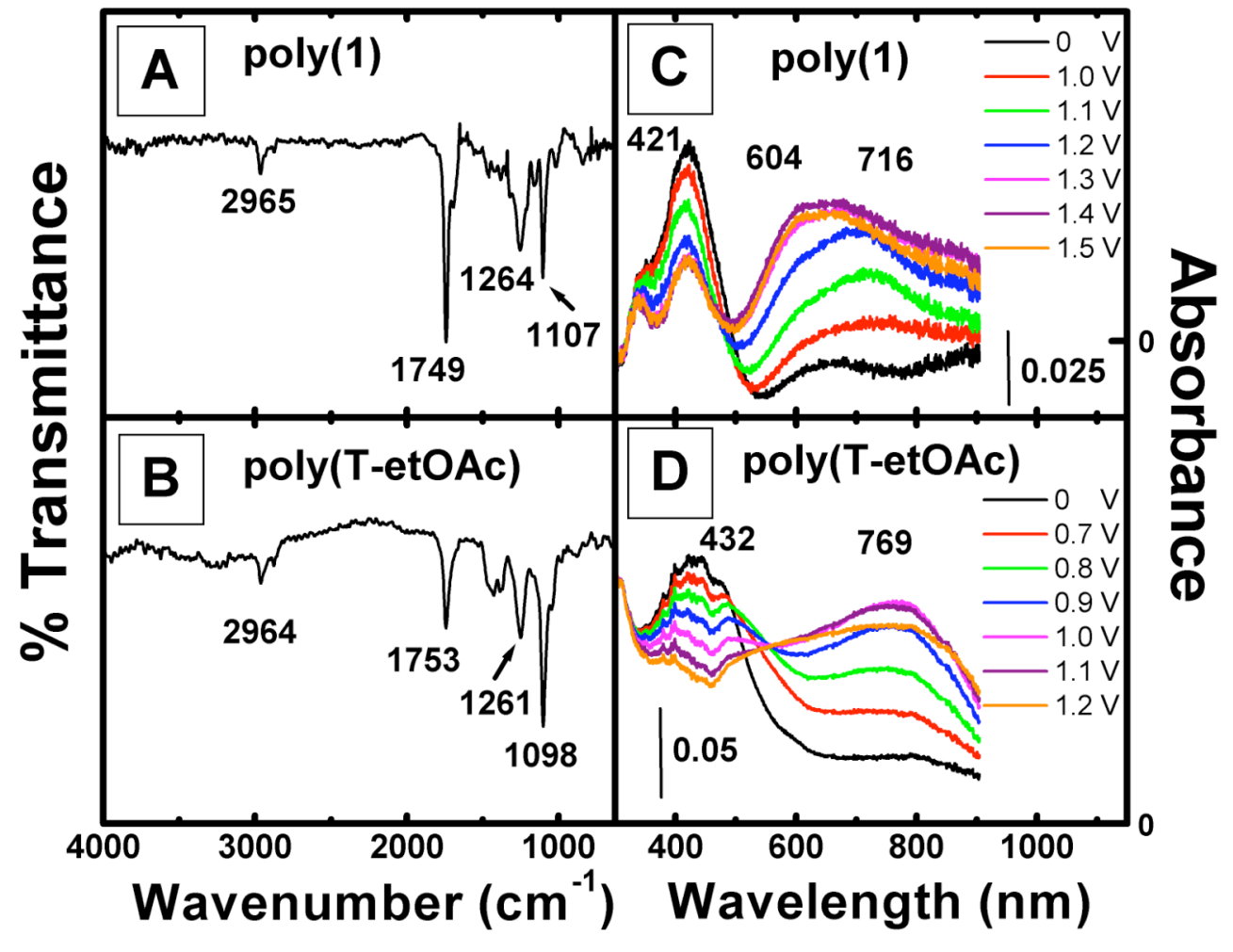

Figure 3. 


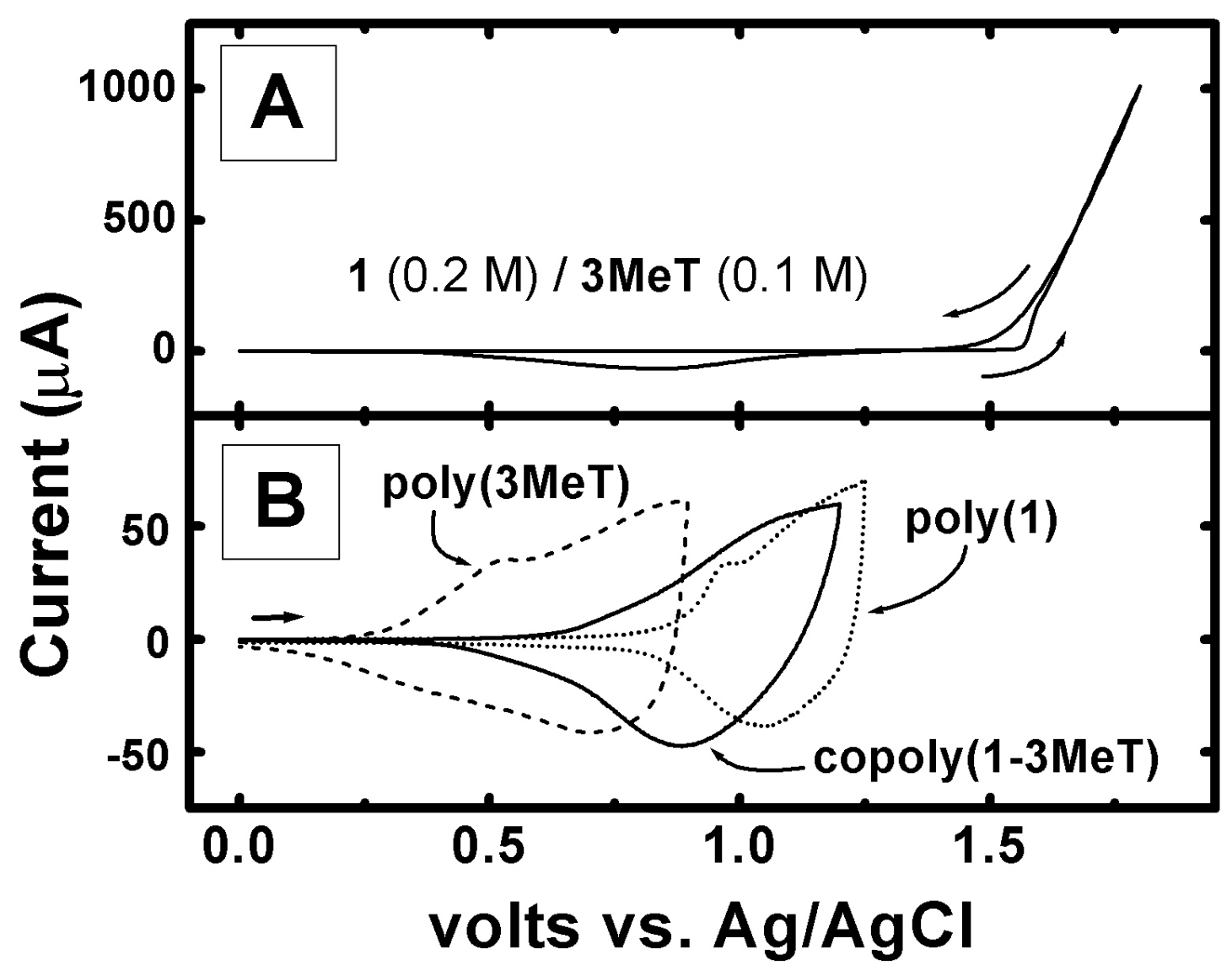

Figure 4. 

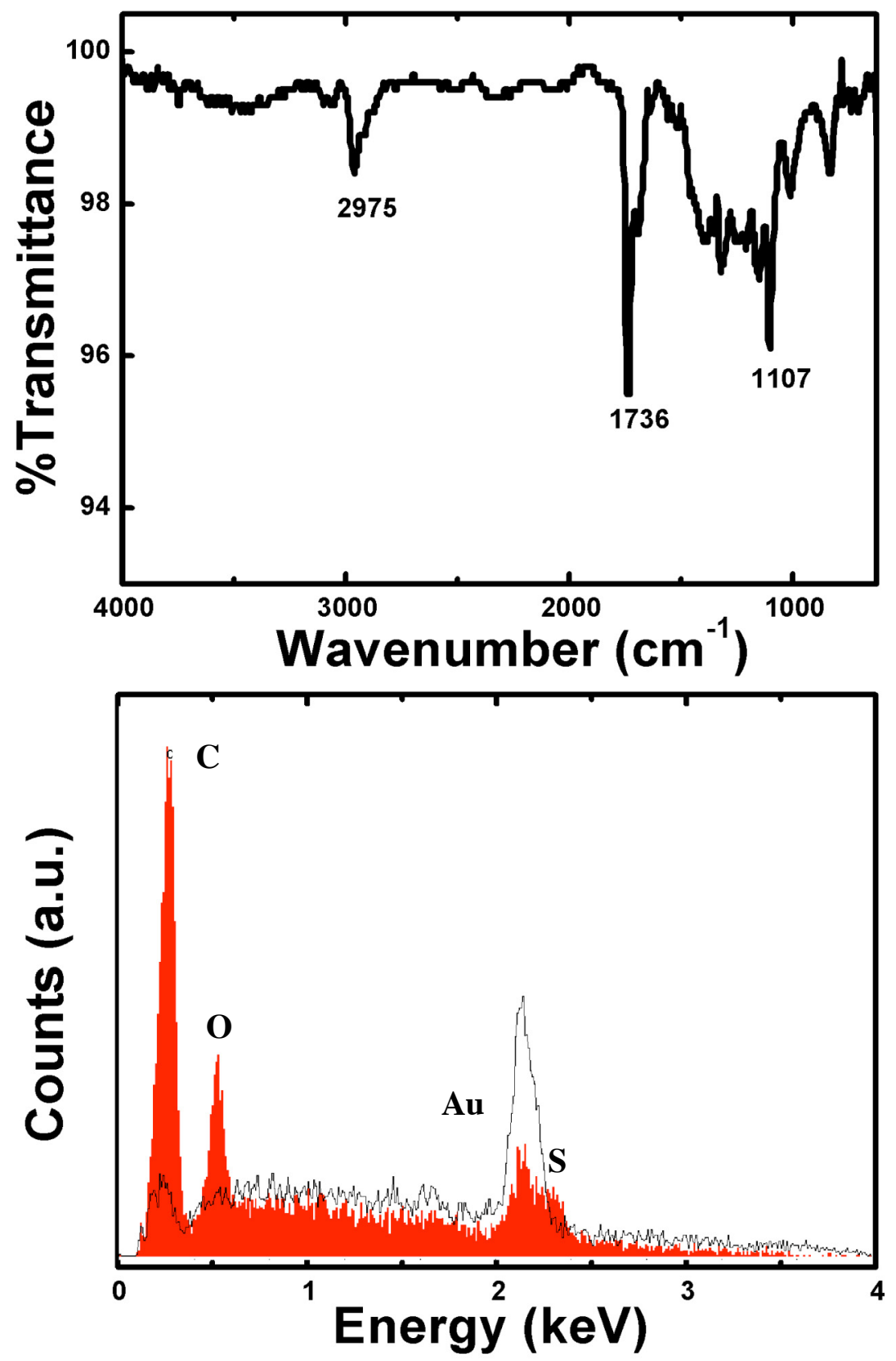

Figure 5. 


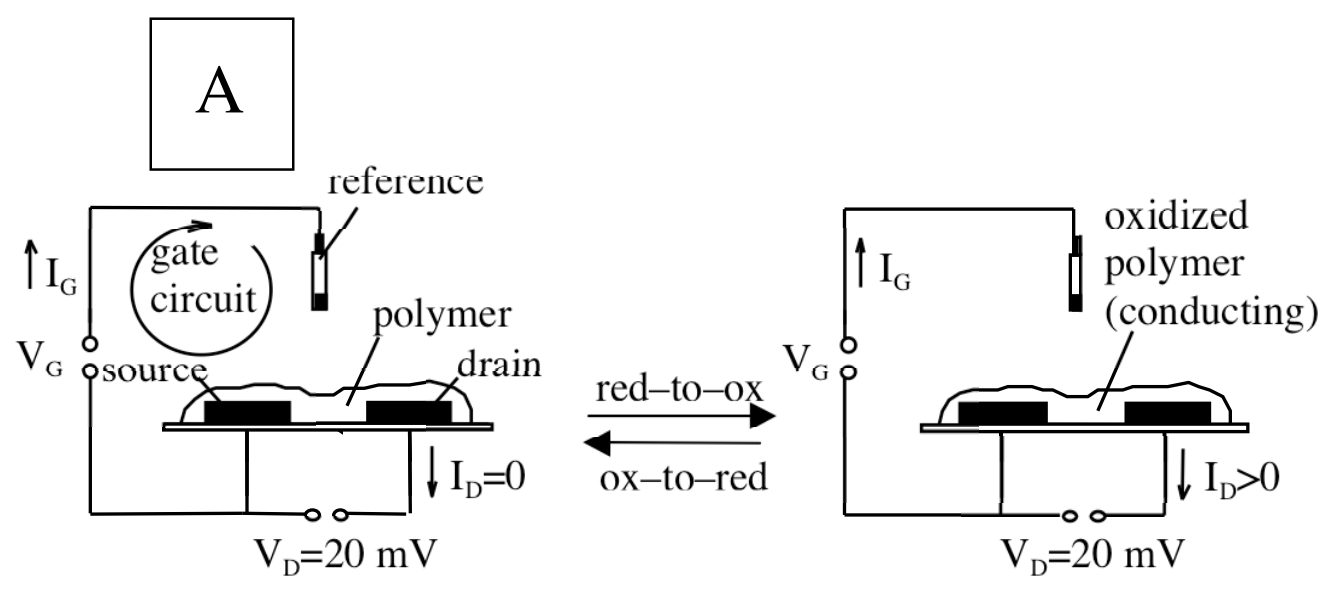

Device "off"

Device "on"

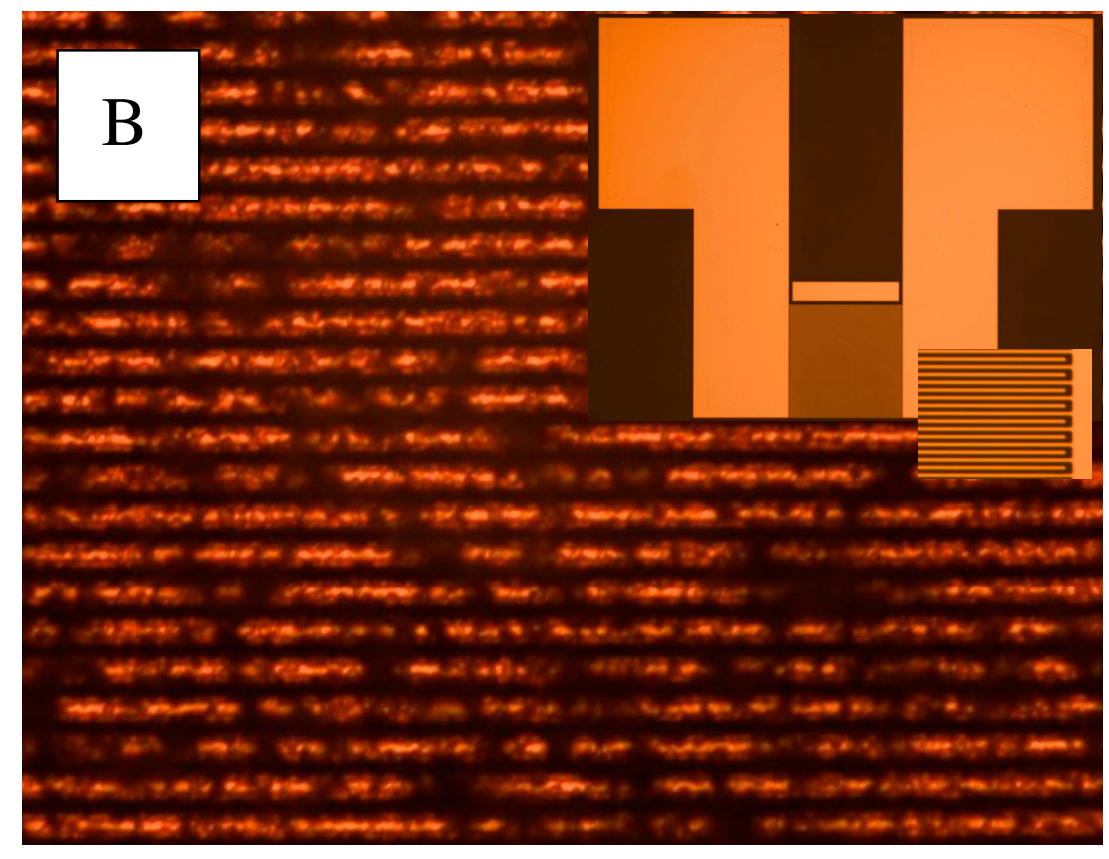

Figure 6. 


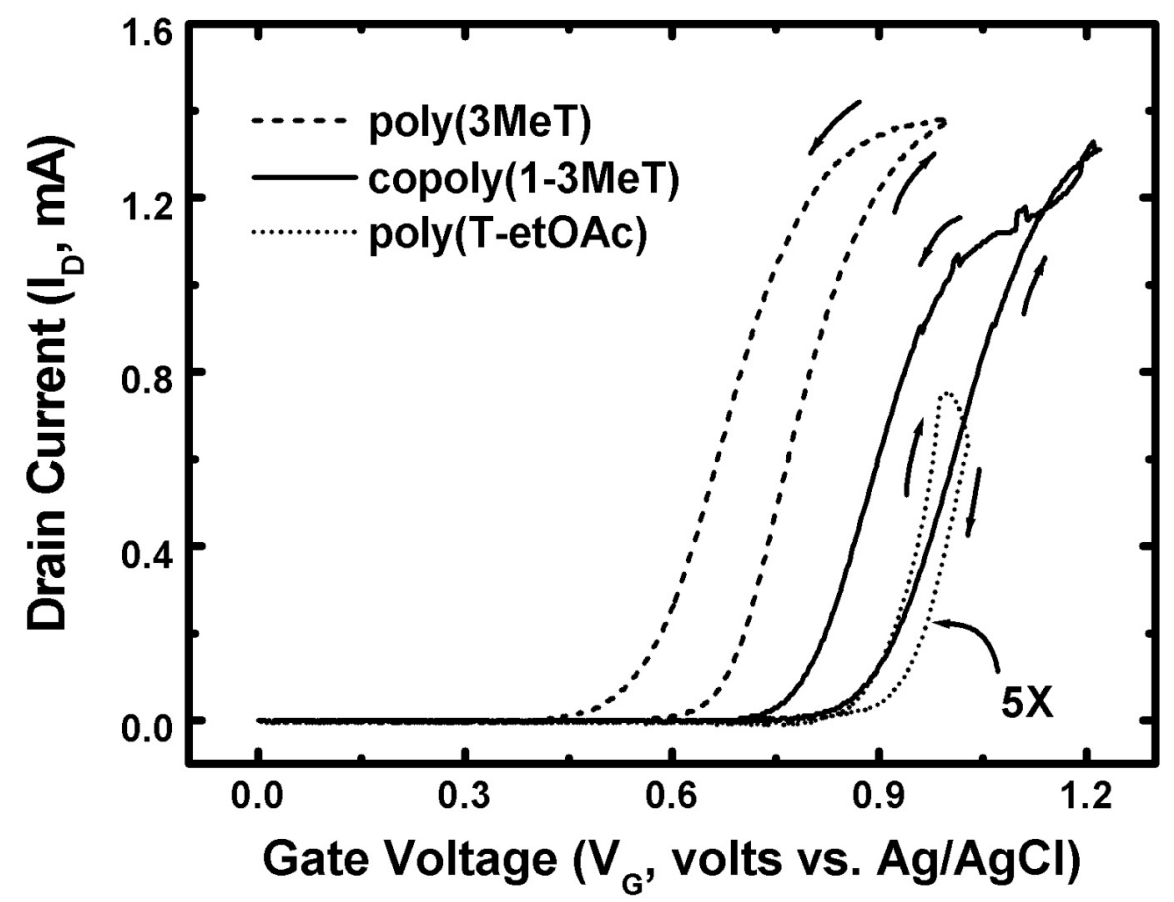

Figure 7. 


\section{References and Notes}

1. Janata, J.; Josowicz, M. Nature Materials 2002, 2, 19-24.

2. (a) Thackeray, J. W.; White, H. S.; Wrighton, M. S. J. Phys. Chem. 1985, 89, 5133-5140. (b) Natan, M.; Wrighton, M. S. Prog. Inorg. Chem. 1989, 37, 391-494. (c) Leventis, N.; Schloh, M. O.; Natan, M. J.; Hickman, J. J.; Wrighton, M. S. Chem. Mater. 1990, 2, 568-576. (d) Swager, T. M. Acc. Chem. Res. 1998, 31, 201-207.

3. Fabrication of functional devices by electrodeposition of the active component at the correct location has been referred to recently as the "grow-in-place" method: Peng, C.-Y.; Kalkan, A. K.; Fonash, S. J.; Gu, B.; Sen, A. Nano Lett. 2005, 5, 439-444.

4. (a) Epstein, A. J.; Ginder, J. M.; Zuo, F.; Woo, H.-S.; Tanner, D. B.; Richter, A. F.; Angelopoulos, M.; Huang, W.-S.; MacDiarmid, A. G. Synthetic Metals 1987, 21, 63-

70. (b) Kohlman, R. S.; Epstein, A. J. in Handbook of Conducting Polymers Second Edition Revised and Expanded, Skotheim, T. A.; Elsenbaumer, R. L.; Reynolds, J. R. Eds; Marcel-Dekker, Inc., New York, N.Y. 1998 Chapter 3, pp 85-121.

5. Kros, A.; Sommerdijk, N. A. J. M.; Nolte, R. J. M. Sensors and Actuators B 2005, 106, 289-295.

6. Roncali, J. Chem. Rev. 1992, 92, 711-738.

7. (a) Welzel, H.-P.; Kossmehl, G.; Schneider, J.; Plieth, W. Macromolecules 1995, 28, 5575-5580. (b) Welzel, H.-P.; Kossmehl, G.; Boettcher, H.; Engelmann, G.; Hunnius, W.-D. Macromolecules 1997, 30, 7419-7426.

8. Qi, Z.; Rees, N. G.; Pickup, P. G. Chem. Mater. 1996, 8, 701-707.

9. Similar issues have been noted with 3-substituents incorporating the $-\mathrm{NH}_{2}$ group; for example, see: Li, G.; Kossmehl, G.; Kautek, W.; Plieth, W.; Zhu, H.; Chan, H. S. O.; Ng, S. C. Macromol. Chem. Phys. 2000, 201, 21-30.

10. (a) Li, G.; Kossmehl, G.; Hunnius, W.; Kautek, H. Z.; Plieth, W.; Melsheimer, J.; Doblhofer, K. Polymer, 2000, 41, 423-432. (b) Bäuerle, P.; Hiller, M.; Scheib, S.; Sokolowski, M.; Umbach, E. Adv. Mater. 1996, 8, 214-218. (c) Hiller, M.; Kranz, C.; Huber, J.; Bäuerle, P.; Schuhmann, W. Adv. Mater. 1996, 8, 219-222. (d) Bäuerle, P.; 
Gaudl, K.-U.; Wurthner, F.; Sariciftci, N. S.; Neugebauer, H.; Mehring, M.; Zhong, C.; Doblhofer, K. Adv. Mater. 1990, 2, 490-494.

11. (a) Rasmussen, S. C.; Pickens, J. C.; Hutchison, J. E. Macromolecules 1998, 31, 933-936. (b) Rasmussen, S. C.; Pickens, J. C.; Hutchison, J. E. Chem. Mater. 1998, 10, 1990-1999. (c) Watson, K. J.; Wolfe, P. S.; Nguyen, S. T.; Zhu, J.; Mirkin, C. A. Macromolecules 2000, 33, 4628-4633. (d) Higgins, T. B.; Mirkin, C. A. Chem. Mater. 1998, 10, 1589-1595. (e) Mouffouk, F.; Brown, S. J.; Demetriou, A. M.; Higgins, S. J.; Nichols, R. J.; Rajapakse, R. M. G.; Reeman, S. J. Mater. Chem. 2005, 15, 1186-1196.

12. Decomposition may come from overoxidation induced by the use of extremely high voltages, over $2.0 \mathrm{~V}$ vs. $\mathrm{Ag} / \mathrm{AgCl}^{7}$

13. Welzel, P.-H.; Kossmehl, G.; Engelmann, G.; Hunnius, W.-D.; Plieth, W. J. Solid State Electrochem. 2001, 5, 141-149.

14. Leventis, N.; Chen, M.; Gao, X.; Canalas, M.; Zhang, P. J. Phys Chem. B 1998, $102,3512-3522$.

15. Leventis, N.; Chen, M.; Liapis, A. I.; Johnson, J. W.; Jain, A. J. Electrochem. Soc. 1998, 145, L55-L58.

16. (a) Asavapiriyanont, S.; Chandler, G. K.; Gunawardena, G. A.; Pletcher, D. J. Electoanal. Chem. 1984, 177, 229-244. (b) Asavapiriyanont, S. Chandler, G. K.; Gunawardena, G. A.; Pletcher, D. J. Electroanal. Chem. 1984, 177, 245-251.

17. Li, G.; Koßmehl, G.; Kautek, W.; Plieth, W.; Melsheimer, J.; Dolbhofer, K.; Hunnius, W.-D.; Zhu, H. Macromol. Chem. Phys. 1999, 200, 450-459.

18. (a) Hill, M. G.; Penneau, J.-F.; Zinger, B.; Mann, K. R.; Miller, L. L. Chem. Mater. 1992, 4, 1106-1113. (b) Zinger, B.; Mann, K. R.; Hill, M. G.; Miller, L. L. Chem. Mater. 1992, 4, 1113-1118. (c) Miller, L. L.; Mann, K. R. Acc. Chem. Res. 1996, 29, 417423. (d) Bäuerle, P.; Segelbacher, U.; Maier, A.; Mehring, M. J. Am. Chem. Soc. 1993, 115, 10217-10223. (e) Audebert, P.; Hapiot, P. Synth. Metals 1995, 75, 95-102.

19. (a) Rabinowitch, E.; Epstein, L. F. J. Am. Chem. Soc. 1941, 63, 69-78. (b) Bergmann, K.; O’Konski, C. T. J. Phys. Chem. 1963, 67, 2169-2171. (c) Mukerjee, P.; Ghosh, A. K. J. Am. Chem. Soc. 1970, 92, 6403-6407. (d) Mukerjee, P.; Ghosh, A. K. J. Am. Chem. Soc. 1970, 92, 6419-6424. (e) Kuwabata, S.; Nakamura, J.; Yoneyama, H. J. 
Electroanal. Chem. Interfacial Electrochem. 1989, 262, 363-373. (f) Leventis, N.; Chen, M. Chem. Mater. 1997, 9, 2621-2631.

20. Thompson, L. A.; Kowalik, J.; Josowicz, M.; Janata, J. J. Am. Chgem. Soc. 2003, $125,324-325$.

21. Groenendaal, L. B.; Jonas, F.; Freitag, D.; Pielartzik, H.; Reynolds, J. R. Adv. Mater. 2000, 12, 481-494.

22. (a) Chung, T. C.; Kaufman, J. H.; Heeger, A. J.; Wudl, F. Phys. Rev. 1984, B30, 702-710. (b) Heeger, A. J.; Kivelson, S.; Schrieffer, J. R.; Su, W. P. Rev. Mod. Phys. 1988, 60, 781-850.

23. An interesting system illustrating the importance of inter-polymer-chain hopping is given in: Lee, D.; Swager, T. M. J. Am. Chem. Soc. 2003, 125, 6870-6871. 